\title{
Nursing Home Integrated Management System Based on ZigBee Technology
}

\author{
Kai-Yuan Meng ${ }^{1, a}$, Xuan-Yu Zhang ${ }^{2, b,{ }^{*}}$, Qing-Nian Cao ${ }^{3, c}$ \\ ${ }^{1}$ School of Computer Science, Xi'an Shiyou University, Xi'an, Shaanxi, 710021, China \\ ${ }^{2}$ School of Computer Science, Xi'an Shiyou University, Xi'an, Shaanxi, 710021, China \\ ${ }^{3}$ School of Computer Science, Xi'an Shiyou University, Xi'an, Shaanxi, 710021, China \\ aemail:kymeng@126.com, bemail:378459175@qq.com, cemail:qncao@126.com
}

Keywords: ZigBee protocol, Management system, Positioning, Nursing home

\begin{abstract}
In recent years, with the degree of the aged phenomenon becoming more serious, the nursing homes are developing rapidly, the scale is expanding and the management also tends to intelligent gradually. For the care and management requirement, a location-help system for the elderly based on ZigBee is designed in this paper, which realized the functions such as inside and outside positioning, falling detection, emergency call and so on. The business management system is developed by using . NET technology, which supports daily business management functions. And integrating the two parts, a set of intelligent nursing home integrated management system has been formed. Application of these functions will effectively improve the management levels and service levels of nursing home, and build a security and humanistic environment to the elderly.
\end{abstract}

\section{Introduction}

In the environment that population ages, the trend of empty nest and family miniaturization continue and disabled elderly population is growing, the nursing and care problems for the elderly become increasingly prominent, institution endowment gradually plays an increasingly important role in the pension mode with the characteristics of management focusing, professional health care services and reducing the pressure of family care. However some problems also expose at the same time, such as potential risk management, security problems, etc. Therefore, studying how to improve the level of management and care and make nursing home tends to informatization and intelligent has great significance.

With the introduction of the Internet of things, current nursing home management system expands the nursing management function on the basis of providing information management platform, meeting the demand of business management, improving the management services level of nursing home. But the solution used for positioning of the domestic excellent system and equipment is based on RFID technology, which has the disadvantages of short distance of signal transmission, expensive, high error rate, not wireless networking and communications data quantity limited. To solve above problems, this paper designs a set of intelligent management system combined with ZigBee technology, which played ZigBee's advantages in location, providing better positioning and help service for old people.

\section{Introduction to ZigBee technology}

ZigBee is a new kind of wireless sensor network technology which has the characteristics of short distance, low speed, low power consumption and high communication efficiency, it is mainly used for wireless communication at close range. ZigBee protocol is mainly composed of five parts: physical layer, medium access control layer, network layer, application layer and security service layer. The communication between layer and layer of ZigBee protocol stack is finished through primitive, most layers provide the data interface Service Access Point and SAP management interface Service Access Point to the upper. The function of data interface is to provide data service 
to the upper, the management interface is to provide internal access, parameters configuration and data management mechanism to the upper.

ZigBee wireless positioning is that a self-organizing network providing the location information of the node through certain methods. The self-organizing network positioning is divided into node self-localization and target positioning, the node self-localization is the process of determining the coordinates of the location of each node, the target positioning is to determine the coordinate of an event or a target within the scope of network coverage. The positioning in this system belongs to target positioning, through arranging network in the known position which are known as the network reference node, to positioning the elderly who carry the target node.

Received Signal Strength Indicator (RSSI) estimates the distance between the nodes through the signal attenuation of the transmission, which is a ranging technology with low power, cheap and widely used, and mainly used in short-range indoor location.

Based on the advantages of low hardware requirements and relatively simple algorithm, the system uses the localization algorithm based on RSSI to determine the elderly's position. The wireless locators compose wireless positioning network in the form of wireless mesh network, to realize signal coverage area. As shown in Fig. 1, when the positioning card move within the network, the communication signal strength between positioning card and each wireless locator will vary with the distance between them, because it has certain corresponding relationship between distance and signal strength, and the coordinates of each wireless locator are known, the positioning card's position can calculated after knowing the signal strength between positioning card and multiple (greater than or equal to 3) wireless locator, which is the location of person using positioning card.

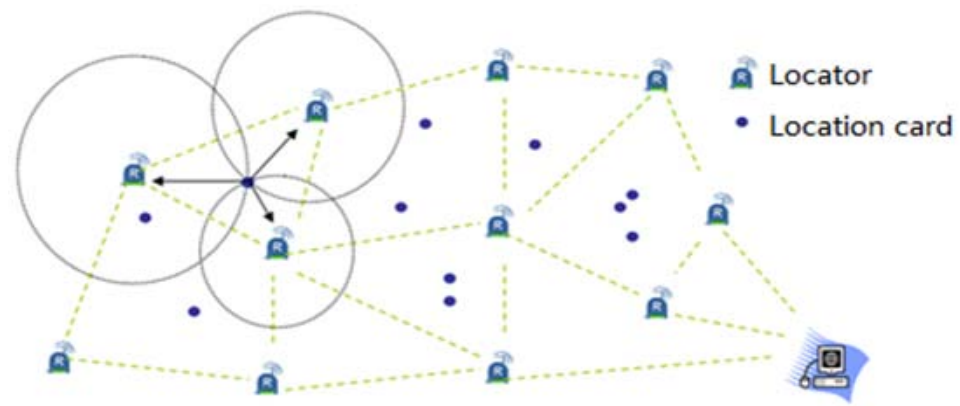

Fig. 1 Positioning principle diagram

\section{Intelligent nursing home integrated management system}

The function setting of nursing home management system must be able to meet the demand of daily management and care, therefore the system function is divided into two broad categories. One is the business management function referenced to traditional nursing management software whit deepening, mining and summarizing, which is used to support the business processes of entire nursing home; The other is care and management function for the elderly in admission and discharge of nursing with the help of the Internet of things technology and the combination of hardware and software. The system's overall framework is shown in Fig. 2. 


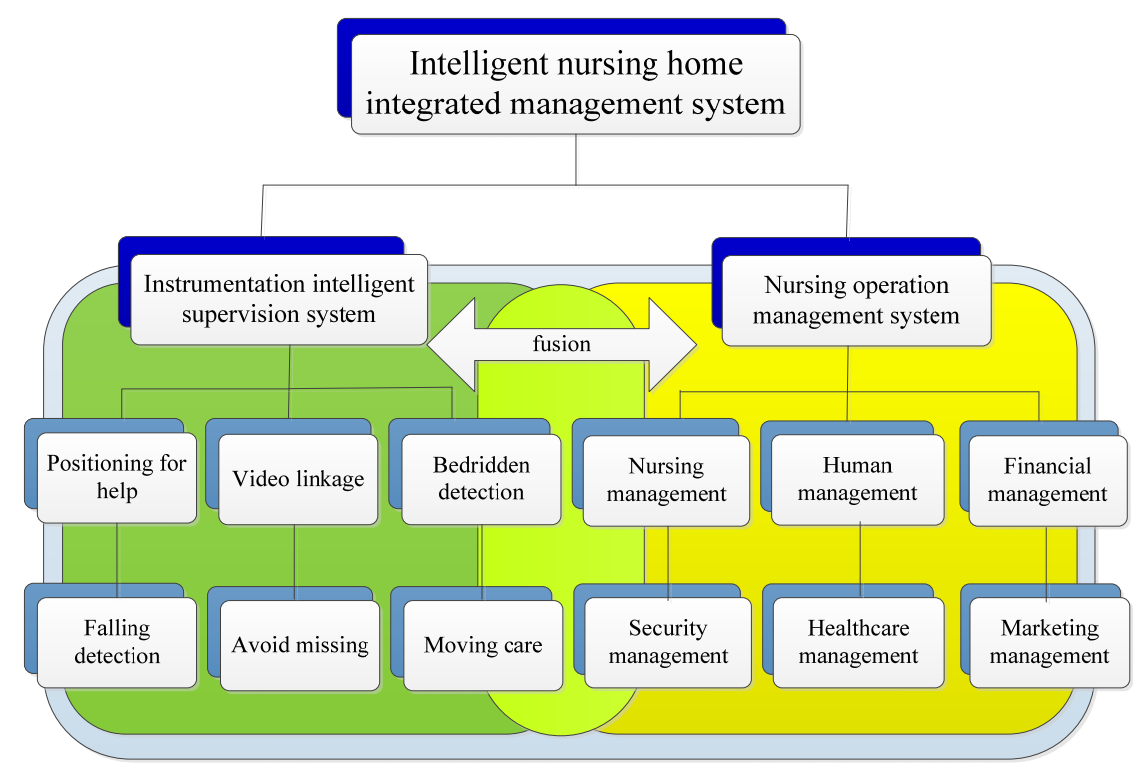

Fig. 2 System overall framework

\section{Positioning and help system}

ZigBee positioning and help system being mainly used to provide all-weather real-time monitoring for the elderly, its main functions include the location tracking, the elderly fall detection, pulse detection, one-button call, night rounds, etc.

(1) Location tracking. The positioning function is mainly to help caregivers to quickly find the position of the specific elderly in the nursing home, realizing real-time indoor positioning.

(2) Fall detection. By monitoring-computer processing the elderly's data and marking down the elderly who fall down, and sending voice prompt to caregivers. The caregivers can respond in the first time, and implement real-time first aid.

(3) One-button call. When the elderly has a demand or emergency case, they can directly contact with nursing service platform, this function improves the level of care by strengthening communication.

(4) Night rounds. The function mainly help nursing home to realize automatical checking at night whether all the elderly have gone to bed, reducing the work burden of nursing staff and improving work efficiency.

Positioning and help system expands the tentacles of nursing home management system and derives the nursing management function of the elderly-centered by introducing positioning card, hardware facilities, wireless locator and wireless gateway in the physical environment.

(1) Positioning card. The card is mainly composed of 5 parts, including ZigBee module, pressure sensors, power switch, lithium batteries and shell, which can continue to send wireless data. The wireless positioner receive packets and send them to PC via Ethernet gateway for monitoring software collecting the real-time location information of wireless positioning label, so as to ensure the location of wireless positioning label. When in an emergency or the old elderly help, they can press the call button at the top of the card, the help information will real time display on the system software, and nursing staff will arrive in time.

(2) Wireless locator. The wireless locator is used to build network signal coverage and receive label report information, and then timely upload it to the wireless positioning gateway to analyse and process. The advanced wireless MESH network architecture is used among locators which can easily achieve a wide range of coverage. There is a large overlapping areas of signal coverage between locator and locator, so without signal vacuum area.

(3) Wireless positioning gateway. The wireless positioning gateway is the collection and processing center of wireless signal of the system, mainly includes wireless communication module, Ethernet communication module and central processing module, the information of all the cards and wireless locator is transfered to the background management software through wireless positioning gateway. The data locator collected is received through wireless communication module and 
transfered to the center processing module to analyze and process, and then forwarded to the LAN via Ethernet communication module.

\section{Business management system}

Business process is mainly divided into 6 functional modules, including reception management, cost management, the elderly management, personnel management, warehouse management and system management.

(1) Reception management. This module provides the viewing and inputting of visiting and receiving record, and the current information of nursing home that might be involved during reception, including data information of the elderly, nursing home beds, the viewing of employee data.

(2) Cost management. Cost management is the heavy part of business management in the system, which includes the settings of various payment amount, and the costing and paying in the whole process of admission to discharge of the elderly.

(3) The elderly management. Which is used to store each elderly's registration information and the service record, old people and their children can easy to understand the elderly's life situation in a nursing home.

(4) Personnel management. Personnel management is a management module set up for nursing home staff, which is used to manage the personnel files and related information.

(5) Warehouse management. This function mainly helps nursing home to manage its stockpiles, timely grasp the latest situation of the work necessary goods and make it convenient to query and purchase for supplies.

(6) System management. System management is mainly used to configure the system software platform, providing the functions of administrator adding and deleting, permissions settings for different administrators, modifying administrator password, default parameter settings for system running, as well as system run log, etc.

\section{System implementation and testing}

In this paper, the implementation of software platform of the nursing home management system based on Internet of things is divided into two parts. One part is the desktop client management software oriented to managerial personnel, which mainly implements the business management functions and the functions collection such as information management, personnel monitoring and personnel positioning in nursing management; The other part provides the Web service, which can make the caregivers, the elderly and children or families, ordinary visitors directly obtain the required information through a browser, as shown in Fig. 3.

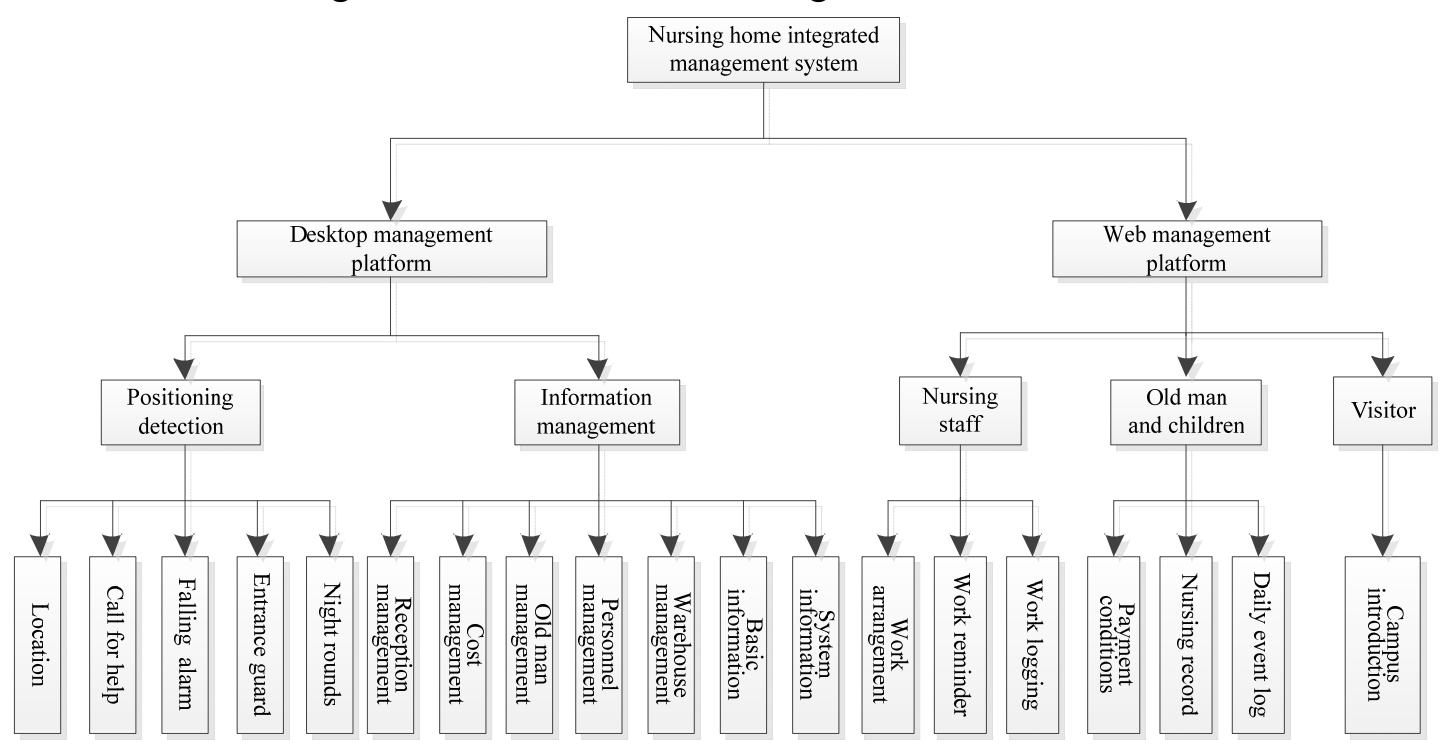

Fig. 3 Software functional diagram of integrated management system 
In order to provide a friendly interactive interface, the functions of desktop management platform is replanned and merged into two subsystems, nosocomial monitoring subsystem and information management subsystem. The monitoring system choice the graphic design in different regions of nursing home as the background, the location of the elderly will be real-time labeled on the plan to implement indoor positioning function to the old. The tags can also help implement other functions at the same time, modifying icon display and increasing the voice prompt can realize the falling down alarm and one-button call function, adding the information on the icon such as name, room number and the pulse can check the state of the elderly in real time. For querying location and night round function, it can be realized by adding the search box and control button in the display. The information management system has little intersection with hardware part, its design focuses on the information input, query and display, implement part mainly includes the interface design, database design and database operation.

The management system uses .NET as software development platform, the coding of software part choose Visual Studio 2010 integrated development environment in .NET framework 4.0. Mainly development language used in the process of software programming is c\#, Html and JavaScript language are used in the Web part. Considering so many transaction in the nursing home need to be processed, Microsoft SQL Server relational database is chosen for data storage. As the desktop management platform to deal with many affairs and frequent data interaction, it uses $\mathrm{C} / \mathrm{S}$ structure model of the fat client design, Web management platform uses a thin client $\mathrm{B} / \mathrm{S}$ structure model, and then the whole system of nursing home management system is built by $\mathrm{C} / \mathrm{S}$ and $\mathrm{B} / \mathrm{S}$ hybrid structure model.

All the primary function menus are listed on the left side of the front page, whose main function modules include real-time call and location service, system user, role permission setting, room state, etc, which will open the corresponding secondary menu if be clicked. The three most commonly used functions are placed in the center of home page — backlog, beds using state and address book.

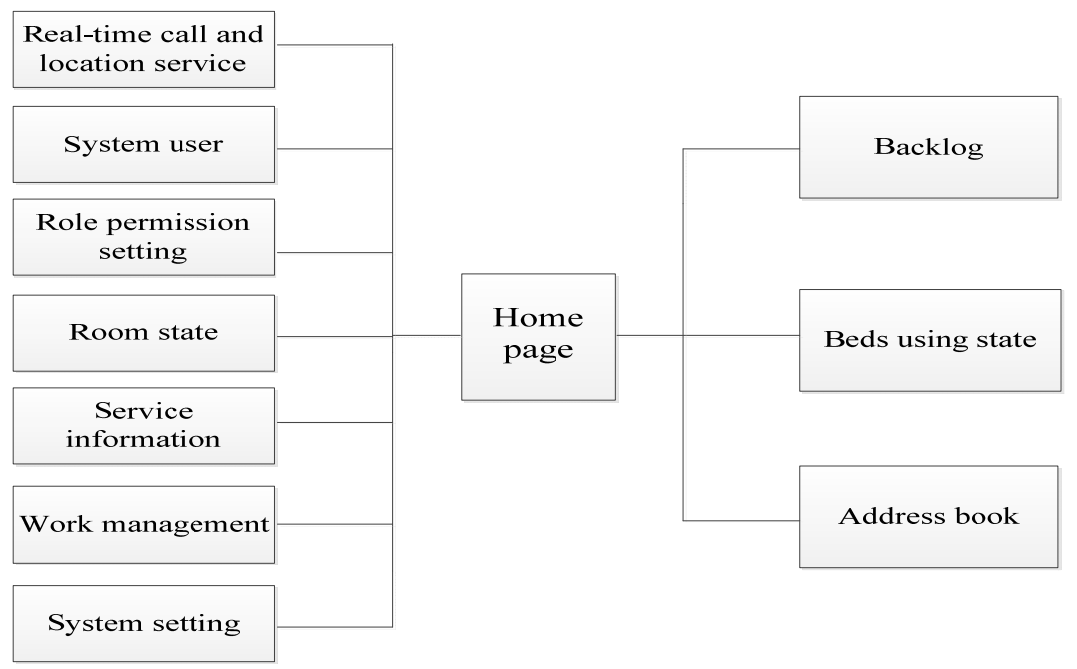

Fig. 4 System home page

\section{Summary}

In this paper, the nursing home integrated management system includes the hardware part which is composed of location card, wireless locator and wireless gateway and the software management platform, which realizes the two big functions of monitoring and information management. The monitoring provides real-time positioning, falling down detection alarm, one-button call and location query to the elderly on campus; The information management provides the support to the business management procedure of nursing home. If the new management system is applied in nursing home, it will provide intelligent management to the nursing home and promote the pension development. 


\section{Acknowledgement}

This research was financially supported by the Shaanxi Provincial Department of education research project (15JK1580) in 2015.

\section{Reference}

[1] Mu Guangzhong, Zhang Tuan, The development trend and strategy of population aging in our country, J. Journal of Huazhong Normal University (Humanities and Social Sciences). 05(2011)29-36.

[2] Fan Xuemei, A survey on development of Internet of Things, J. Computer Measurement \& Control. 05(2011)1002-1004.

[3] Zhou Yan, Study of wireless sensor network localization algorithm based on RSSI, J. Computer Science. 2009(04).

[4] Meng Lin, Research on community medical management system based on ZigBee technology and long-range communication technology, Jilin University, 2009.

[5] Ma Gang, Design and implementation of the underground mine personnel location and tracking system based on ZigBee, Dalian University of Technology, 2008.

[6] Wang Qin, Research and implementation of wireless sensor network based on ZigBee, J. Journal of Chongqing University of Technology (Natural Science). 25(2011). 